\title{
A Modular Approach to Multi-Robot Control
}

\author{
Robert J. Anderson \\ Sandia National Labs \\ M.S. 1176, P.O. Box 5800 \\ Albuquerque, NM 87185 \\ bjander@isrc.sandia.gov
}

\section{Abstract}

The ability to rapidly command multi-robot behavior is crucial for the acceptance and effective utilization of multiple robot control. To achieve this, a modular multiple robot control solution is being pursued using the SMART modular control architecture [1]. This paper investigates the development of a new dual-arm kinematics module (DUAL_KIN) which allows multiple robots, previously controlled as separate stand-alone systems, to be controlled as a coordinated multi-robot system. The DUAL_KIN module maps velocity and force information from a center point of interest on a grasped object to the tool centers of each grasping robot. Three-port network equations are used and mapped into the scattering operator domain in a computationally efficient form. Application examples of the DUAL_KIN module in multi-robot coordinated control are given.

\section{Introduction}

Industrial robots conduct most part handling operations using single robot arms. If the manipulated objects are large, then care is taken to lift an object at its center of mass, or if that is not possible, then a larger robot is chosen to conduct the operation. Humans, on the other hand, rarely try to manipulate a large object with a single hand; instead, humans naturally use two hands to deal with the uncertainty in the center of mass location, to provide better orientation control, and to distribute the payload. If the object is still too large, then more (or stronger) people are asked to lend a hand.

Researchers have long known how to coordinate the motion of multiple robots ([5] [6] [7] [8] [10] [11] [12] [13]). They have developed equations for resolving intetnal forces, for providing sensor-based adjustment, and for coordinating the sequencing of motions. Yet there has been little acceptance of multiple robot control techniques $i$ in industry.

The main impediment in utilizing multiple robots is not a lack of useful theory, but the need for better programming methodologies. It is difficult to program multiple robot behavior. In a multiple robot workcell, robots

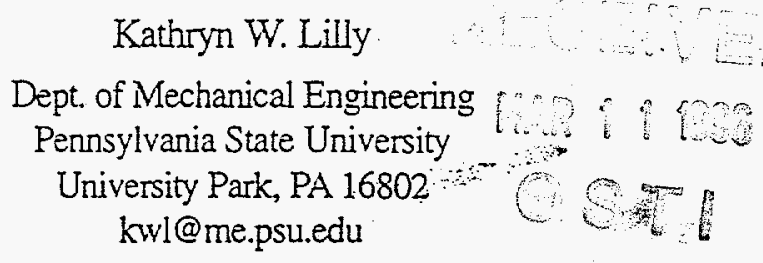

perform tasks in many different modes. They perform autonomously, making sure not to collide with other robots while sharing resources such as tooling or part bins. They perform in a cooperating fashion, where one robot fixtures a part, holds a camera, or transfers a small object for another robot. Robots may also perform in a coordinated fashion, where the motion of all robots must be carefully sequenced. The robot programmer must be able to move seamlessly between these different modes. of behavior, using different combinations of two or more robots.

Our approach to simplifying the programming of multiple robot arms has three components: (1) a task sequence controller; (2) a graphical programming interface; and (3) a low-level modular real-time interface. The task sequence controller provides a text-based interface which describes the state diagram of events required for a robot system to conduct a given task. This component is currently under development. The graphical programming interface allows the operator to plan and display motion using "tag-points" in a 3-dimensional graphical mock-up of the multi-arm workcell. The programmer accesses different functionality in a windowing environment, and associates motion with the objects to be manipulated in the environment. This interface is further described in [9].

The third component of our approach, the low-level modular real-time interface, is the subject of this paper. The interface is based on the SMART (Sequential Modular Architecture for Robotics and Teleoperation) robot control system ([1] [2] [3]). SMART allows the operator to describe robot behavioral modes in terms of graphical icons. Each icon represents a subnetwork in the complete robot system network. The user combines icons representing input devices, sensors, kinematics, simulated dynamics, constraints, and actual robot hardware to create a working system.

To date, the SMART system has been applied to numerous telerobotic systems, all of which use only one robot. The corresponding SMART networks have consisted solely of one-port and two-port elements. In this paper, we describe the development of a new SMART module which allows us to interconnect an arbitrary number of robot devices. Combinations of these and other

This work was suponted by he United

Slaies Depemmen of Energy under

Contract DE-ACO4-94A195000. 


\section{DISCLAMMIER}

Portions of this document may be illegible in electronic image products. Images are produced from the best available original document. 


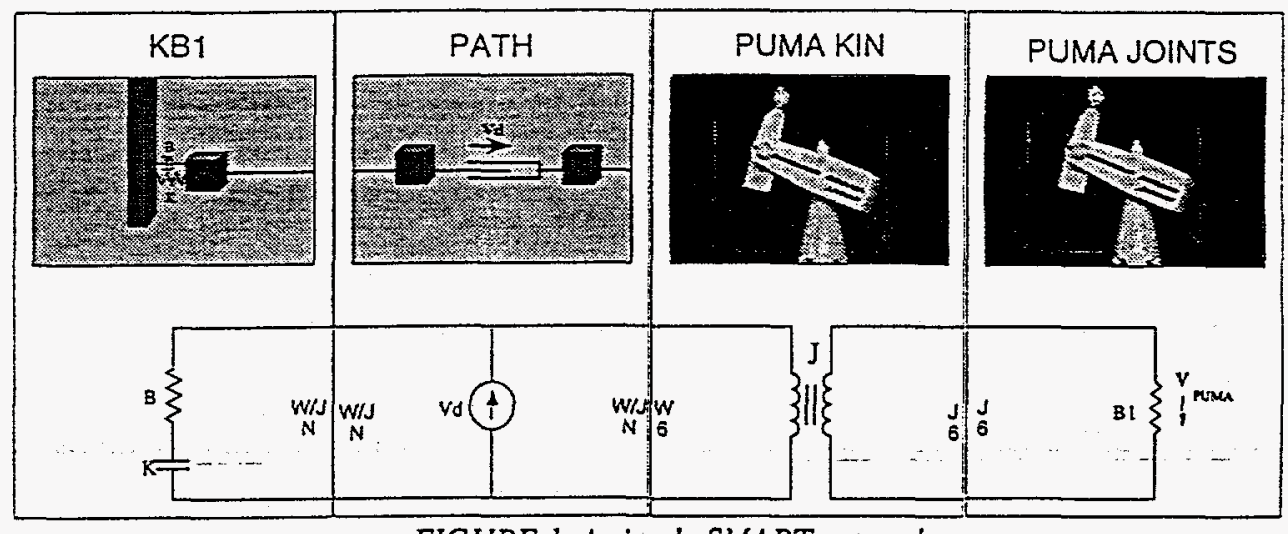

FIGURE 1. A simple SMART network.

rapidly reconnected and swapped in and out without inducing instability.

The scattering operator, i.e., the mapping between input and output wave variables, has a particularly pleasing form. Its norm represents the energy gain for the network. Assuming that the underlying network is passive, the scattering operator should always have a norm which is less than or equal to one. This implies that the output waves $\left(b_{i}\right)$ should always have a norm less than or equal to the input waves $\left(a_{i}\right)$. If the port is lossless, then the associated scattering operator will have a unity norm. This feature enables the freedom in sampling mentioned above, since a delayed signal has the same norm as the original signal, and thus, a sample-induced time delay will not affect the passivity of the network [3].

The goal, then, is to represent every subnetwork module/port in a scattering operator form. Once this is accomplished, we can arbitrarily distribute, sample, and update these ports using the SMART software environment.

Currently, over a hundred different SMART modules have been developed. For example, modules exist for generating trajectories (PATH module), for mapping robot kinematics from the world frame into the joint frame (e.g. PUMA_KIN and PAR_KIN modules), for filtering and terminating a network (e.g. KB1 module), and for controlling robot motion in joint space (e.g. PUMA_JOINTS and PAR_JOINTS modules). A simple SMART grid using these modules to create an autonomous world space motion controller for a PUMA robot is shown in Figure 1.

Our goal here is to develop a new module which will accept position and orientation commands for a rigid body grasped by two robots and compute the corresponding position and velocity at the two grasped points on the rigid body. This module must also convey force/torque information arising from local force sensors, kinematic limitations, or imposed constraints for each grasping robot, and transform these forces into the grasped frame.
Once this module is developed in a readily computable form, it can be combined with existing modules to create different multi-robot behaviors.

\section{Smart module for two-arm control}

\subsection{Derivation of the force and yelocity equations}

The first stage in developing a new SMART module is to characterize the module, i.e., the network port, in terms of force and velocity equations. Our focus here is on the mapping between force and velocity at a center point of interest on a single rigid body to the corresponding forces and velocities at two grasp locations on the same rigid body.

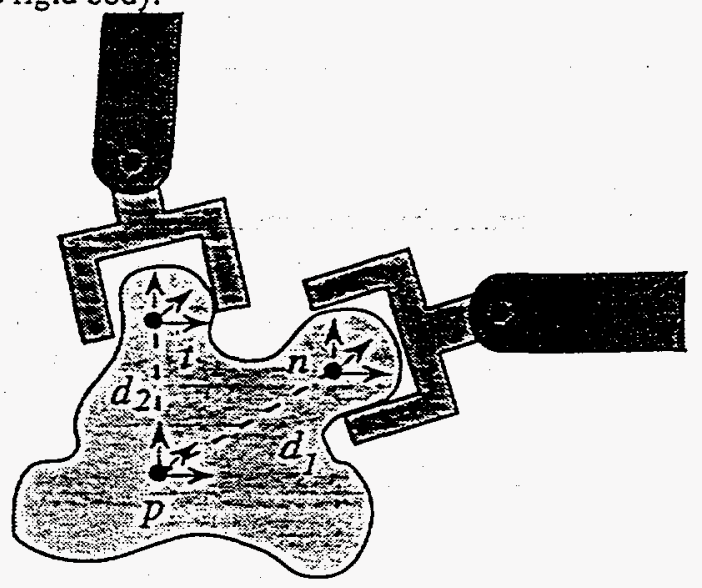

FIGURE 2. Two arms grasping a rigid object.

Consider the system shown in Figure 2. In this figure, two robots grasp a common rigid object at points $n$ and $t$, respectively'. The robot system operator, on the

1. First subscripts $p, n$, and $t$ correspond to previous, next and top, which correspond to the ports used in a SMART network (see Figure 3). Second subscripts, $p$ and $r$, refer to the three positioning degrees of freedom and the three rotational degrees of freedom, respectively. 


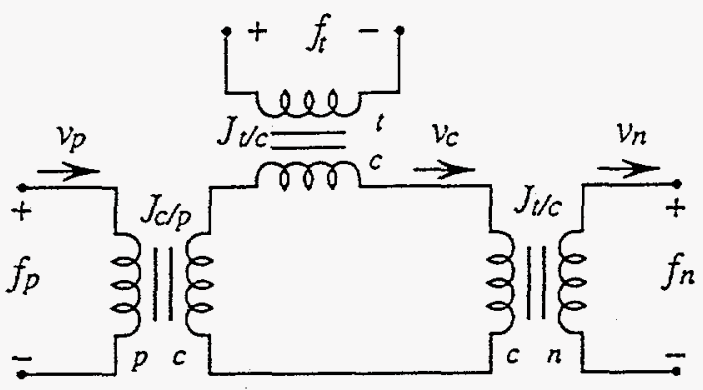

FIGURE 5. Network for two-arm grasp with center point.

The corresponding Jacobians are defined as follows:

$$
\begin{gathered}
J_{p / c}=\left[\begin{array}{cc}
I_{3} & X\left(d_{p}\right) \\
0_{3} & I_{3}
\end{array}\right], J_{t / c}=\left[\begin{array}{cc}
I_{3} & X\left(d_{t}\right) \\
0_{3} & I_{3}
\end{array}\right] \\
J_{n / c}=\left[\begin{array}{cc}
I_{3} X\left(d_{n}\right) \\
0_{3} & I_{3}
\end{array}\right]
\end{gathered}
$$

and the force and velocity mappings are given by:

$$
\begin{aligned}
v_{n} & =J_{n / c} \nu_{c}=J_{n / c} J_{p / c}^{-1} \nu_{p} \\
v_{t} & =J_{t / c \nu_{c}}=J_{t / c} J_{p / c}^{-1} \nu_{p} \\
f_{p} & =J_{p / c}^{-T}\left(J_{n / c}^{T} f_{n}+J_{t / c}^{T} f_{t}\right)
\end{aligned}
$$

This network appears to be more complicated than the original network (Figure 3), but it has the same input-output behavior, where $J_{n / p}=J_{c / n}^{-1} J_{c / p}$ and $J_{v^{\prime}}=J_{c / t}^{-1} J_{c / p \text {. It }}$ also results in a much simpler representation of the scattering operator.

\subsection{Derivation of the scattering operator}

The scattering operator mapping for the three-port network shown in Figure 5 is defined by the following equation:

$$
\begin{gathered}
{\left[\begin{array}{l}
b_{p} \\
b_{n} \\
b_{t}
\end{array}\right]=\left[\begin{array}{c}
f_{p}-Z_{0} v_{p} \\
f_{n}+Z_{0} v_{n} \\
f_{t}+Z_{0} v_{t}
\end{array}\right]=S_{p \times n \times t}\left[\begin{array}{l}
f_{p}+Z_{0} v_{p} \\
f_{n}-Z_{0} v_{n} \\
f_{t}-Z_{0} v_{t}
\end{array}\right]} \\
=S_{p \times n \times t}\left[\begin{array}{l}
a_{p} \\
a_{n} \\
a_{t}
\end{array}\right]
\end{gathered}
$$

\footnotetext{
which yields the following scattering operator:
}

$S_{p \times n \times t}=\left[\begin{array}{ccc}I_{\sigma}-J_{p / c} D_{c} J_{p / c}^{T} & J_{p / c} D_{c} J_{n / c}^{T} & J_{p / c} D_{c} J_{t / c}^{T} \\ J_{n / c} D_{c} J_{p / c}^{T} & I_{\sigma}-J_{n / c} D_{c} J_{n / c}^{T} & -J_{n / c} D_{c} J_{t / c}^{T} \\ J_{t / c} D_{c} J_{p / c}^{T} & -J_{t / c} D_{c} J_{n / c}^{T} & I_{6}-J_{t / c} D_{c} J_{t / c}^{T}\end{array}\right]$

where:

$$
\begin{aligned}
& D_{c}=2\left(J_{p / c}^{T} J_{p / c}+J_{n / c}^{T} J_{n / c}+J_{t / c}^{T} J_{s / c}\right)^{-1} \\
& =2\left(\left[\begin{array}{ll}
I_{3} & 0_{3} \\
X_{p}^{T} & I_{3}
\end{array}\right]\left[\begin{array}{ll}
I_{3} & X_{p} \\
I_{3} & I_{3}
\end{array}\right]+\left[\begin{array}{ll}
I_{3} & O_{3} \\
X_{n}^{T} & I_{3}
\end{array}\right]\left[\begin{array}{ll}
I_{3} & X_{n} \\
O_{3} & I_{3}
\end{array}\right]+\left[\begin{array}{ll}
I_{3} & 0_{3} \\
X_{t}^{T} & I_{3}
\end{array}\right]\left[\begin{array}{ll}
I_{3} & X_{t} \\
O_{3} & I_{3}
\end{array}\right]\right)^{-1} \\
& =2\left(\left[\begin{array}{cc}
I_{3} & X_{p} \\
X_{p}^{T} & I_{3}+X_{p}^{T} X_{p}
\end{array}\right]+\left[\begin{array}{cc}
I_{3} & X_{n} \\
X_{n}^{T} & I_{3}+X_{n}^{T} X_{n}
\end{array}\right]+\left[\begin{array}{cc}
I_{3} & X_{p} \\
X_{t}^{T} & I_{3}+X_{t}^{T} X_{t}
\end{array}\right]\right)^{-1} \\
& =2\left[\begin{array}{c}
3 I_{3} \\
\left(X_{p}+X_{n}+X_{t}\right)^{T} 3 I_{3}+X_{p}^{T} X_{p}+X_{n}^{T} X_{n}+X_{t}^{T} X_{t}
\end{array}\right]^{-1}
\end{aligned}
$$

The additional frame defined at point $c$ allows us to simplify this expression, since, by definition:

$$
\begin{aligned}
& X_{p}+X_{n}+X_{t}=X\left(d_{p}\right)+X\left(d_{n}\right)+X\left(d_{t}\right) \\
& =X\left(d_{p}+d_{n}+d_{t}\right) \\
& =X\left(x_{p p}-x_{c p}+x_{n p}-x_{c p}+x_{t p}-x_{c p}\right) \\
& =X\left(0_{3 / 1}\right) \\
& =0_{3}
\end{aligned}
$$

Thus, we may write:

$$
\begin{aligned}
D_{c} & =2\left[\begin{array}{cc}
3 I_{3} & 0_{3} \\
0_{3} & 3 I_{3}+X_{p}^{T} X_{p}+X_{n}^{T} X_{n}+X_{l}^{T} X_{3}
\end{array}\right]^{-1} \\
& =\left[\begin{array}{cc}
\left(\frac{2}{3}\right) I_{3} & 0_{3} \\
0_{3} & D_{c r}
\end{array}\right]
\end{aligned}
$$

with $D_{c r}=2\left(3 I_{3}+X_{p}^{T} X_{p}+X_{n}^{T} X_{n}+X_{t}^{T} X_{t}\right)^{-1}$.

If the additional center frame is not defined at point $c$, then the block diagonal form of $D_{c}$ is not evident, and the computation of the scattering operator requires a six dimensional matrix inverse, rather than a three dimensional matrix inverse. Substituting Eq. 18 into Eq. 15 
frame. The ROBOT_KIN module, which maps quantities from world space to joint space, assumes that its input position and velocity are given with respect to its own coordinate system. Thus, for a two-robot grasp, there may be three reference frames of interest: a universal reference frame, and the local reference frames of the two robots. These frames will typically differ by a fixed translation and a fixed orientation. The DUAL_KIN module being developed here must accommodate these different reference frames. To do so, another scattering operator computation step is introduced.

Assume that the input velocity to the port/module, $v_{p}$, is represented in a universal reference frame. Then the robot velocity, $v_{n}$, derived from the scattering operator, will also be in the world frame. Let frame $n$ ' represent the same position/orientation of the robot expressed in the robot's base coordinate frame. Then the position of the robot attached to the next port, $x_{n}$, and its orientation, $R_{n}$, may be written as follows:

$$
\begin{gathered}
x_{n^{\prime}}=R_{n^{\prime} / n^{2}} x_{n}+d_{n^{\prime} / n} \\
R_{n^{\prime}}=R_{n^{\prime} / n^{\prime}} R_{n}
\end{gathered}
$$

where $R_{n} / n$ represents the fixed orientation between the next robot's frame and the universal frame, and $d_{n} / n$ is the fixed distance between the two frame origins. The force and velocity mappings between these two frames are thus given by:

$$
v_{n^{\prime}}=\left[\begin{array}{c}
v_{n p^{\prime}} \\
v_{n r^{\prime}}
\end{array}\right]=\left[\begin{array}{cc}
R_{n^{\prime} / n} & O_{3} \\
O_{3} & R_{n^{\prime} / n}
\end{array}\right]\left[\begin{array}{l}
v_{n p} \\
v_{n r}
\end{array}\right]=J_{n^{\prime} / n^{\prime}} v_{n}
$$

and:

$f_{n}=\left[\begin{array}{c}f_{n p} \\ f_{n F}\end{array}\right]=\left[\begin{array}{cc}R_{n^{\prime} / n}^{T} & O_{3} \\ O_{3} & R_{n^{\prime} / n}^{T}\end{array}\right]\left[\begin{array}{c}f_{n p^{\prime}} \\ f_{n r^{\prime}}\end{array}\right]=J_{n^{\prime} / n}^{T} f_{n^{\prime}}(28)$

The resulting scattering operator for this two-port mapping is defined by:

$$
\begin{gathered}
{\left[\begin{array}{c}
b 1_{n} \\
b_{n^{\prime}}
\end{array}\right]=\left[\begin{array}{c}
f_{n}-Z_{0^{\prime}} v_{n} \\
f_{n^{\prime}}+Z_{0^{\prime} n^{\prime}}
\end{array}\right]=\left[\begin{array}{rr}
O_{6} & J_{n^{\prime} / n}^{T} \\
J_{n^{\prime} / n} & O_{6}
\end{array}\right]\left[\begin{array}{r}
f_{n}+Z_{0} v_{n} \\
f_{n^{\prime}}-Z_{0^{\prime}} v_{n^{\prime}}
\end{array}\right]} \\
=S_{n^{\prime} \times n}\left[\begin{array}{c}
a_{n} \\
a_{n^{\prime}}
\end{array}\right]
\end{gathered}
$$

which can be decomposed into two independent equations as follows:

$$
\begin{aligned}
& b 1_{n}=J_{n^{\prime} / n^{2} n^{\prime}}^{T} \\
& b_{n^{\prime}}=J_{n^{\prime} / n^{\prime}} a 1_{n}
\end{aligned}
$$

This same procedure can be followed for the robot connected to the top of the three-port module to compute the scattering operator, $S_{t} x_{b}$ as well as mappings for $a l_{t}$ and $a_{t^{\prime}}$ (to $b_{t}$, and $b 1_{t}$, respectively). Once these two scattering operators are obtained, the final algorithm for updating the wave variables for the DUAL_KIN module consists of the following five steps:

1. Receive wave inputs, $a_{n}$. and $a_{t^{\prime}}$, from the next and top ports and compute output waves $b 1_{n}$ and $b 1_{t}$ as shown in Eq. 30.

2. Equate $a_{n}=b 1_{n}$ and $a_{t}=b 1_{t}$.

3. Receive the new input wave, $a_{p}$, and compute the three-port scattering operation (Eq. 23) to get new values for $b_{n}$ and $b_{l}$, and to compute the output wave, $b_{p}$.

4. Equate $a 1_{n}=b_{n}$ and $a 1_{t}=b_{l}$.

5. Compute the output waves, $b_{n}$. and $b_{t}$, as shown in Eq. 31.

In addition to passing wave variables to close force and velocity feedback loops, a SMART module must pass position variables forward. The complete position mapping for the DUAL_KIN module is given by:

$$
\begin{aligned}
& x_{n^{\prime}}=R_{n^{\prime} / n}\left(x_{p p}+R_{p r^{\prime}} d_{1}\right)+d_{n^{\prime} / n} \\
& R_{n^{\prime}}=R_{n^{\prime} / n} R_{p r} \\
& x_{t^{\prime}}=R_{t^{\prime} / l}\left(x_{p p}+R_{p r^{\prime}} d_{2}\right)+d_{t^{\prime} / t} \\
& R_{t^{\prime}}=R_{i^{\prime} / t} R_{p r}
\end{aligned}
$$

\section{Implementation issues}

Having derived the scattering operator equations and the position mapping for the DUAL_KIN module, a number of issues still need to be resolved to complete the module development. A SMART module requires three routines: (1) an initialization routine; (2) a filter constant setting routine; and (3) an update toutine.

For the DUAL_KN module, the initialization routine is trivial, since no hardware needs to be initialized before the module is executed. The initialization routine must simply call the filter constant setting routine with default filter constants in the event that the user has not predefined the filter constants.

The "filter constants" for the DUAI_KIN module consist of the center of rotation of the grasped object, $x_{p p}$, 


\section{Experimental results}

The Sandia National Laboratory multi-arm research facility consists of two overhead $\mathrm{PaR}$ 6-DOF gantry robots sharing a common run-way, two PUMA 760 robots, and on occasion, other smaller robots such as the PUMA 560. The two $P a R$ robots have the additional feature of a removable 2-axis wrist, which allows each of them to be converted from a 6 -axis robot to a 4-axis robot with a $1500 \mathrm{lb}$. lift capacity. Shared robot behaviors may be conducted by any two, any three, or all four of these robots. The robots are currently being applied to largescale decommissioning projects, in which the gantry robots conduct large scale part removal.

At the 1995 DOE Robotics Forum, held in Albuquerque, the DUAL_KNN module developed in this paper was demonstrated in the multi-arm laboratory using two PUMA 760's and one of the PaR robots. Eleven different behavioral modes were used to conduct the demonstration. Included in these were three different shared modes of operation involving each pair-wise combination of robots and one mode showing the combined behavior of all three robots. The system was shown to move seamlessly between these different modes of operation in order to conduct both teleoperated and autonomous operations. The bilateral transmission of force information was shown to prevent overdriving the grasped object past robot singularities and joint limits. No stability problems occurred in any behavioral mode.

\section{Conclusions}

In this paper, we describe a method to rapidly prototype and command multi-robot behavior in a modular fashion. The key to this approach is the development of a new SMART module called the DUAL_KIN module which allows branching of the command input to two different robot devices. When connected in series, this same module may be used to control three or more robots. When combined with existing SMART modules for trajectory path generation, robot kinematics, and robot joint commands, a complete multi-robot control system is obtained.

The key to developing a SMART module lies in the ability to derive a scattering operator mapping between input and output wave variables in an efficient computable form. This was done for the DUAL_KIN module in a way which accommodates the different local coordinate systems of each robot in the multi-robot system. Details of the input-output wave mapping were given, as well as the synchronization method for the module.

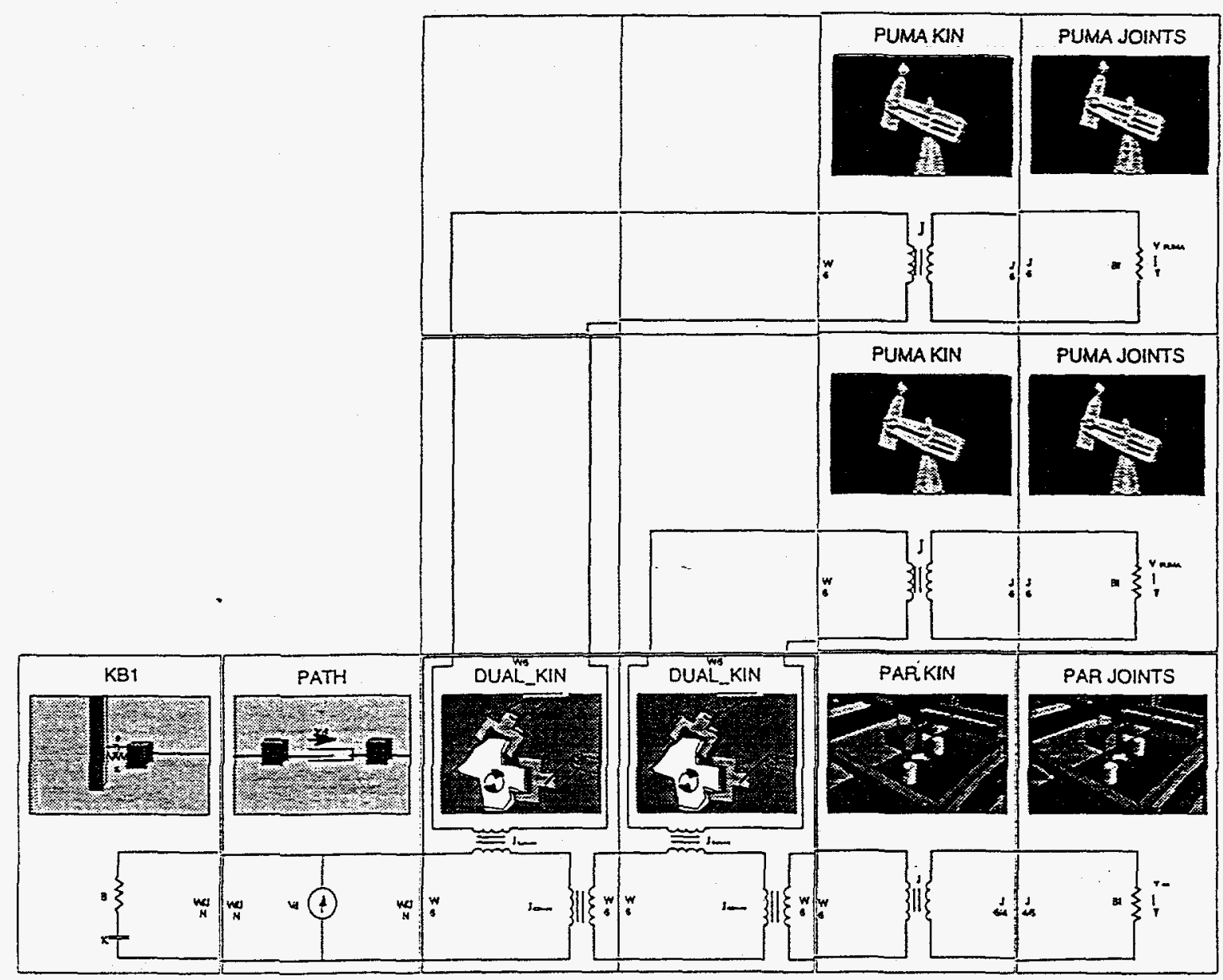

FIGURE 7. Using the DUAL_KIN module for three-arm coordination. 


\section{DISCLAIMER}

This report was prepared as an account of work sponsored by an agency of the United States Government. Neither the United States Government nor any agency thereof, nor any of their employees, makes any warranty, express or implied, or assumes any legal liability or responsibility for the accuracy, completeness, or usefulness of any information, apparatus, product, or process disclosed, or represents that its use would not infringe privately owned rights. Reference herein to any specific commercial product, process, or service by trade name, trademark, manufacturer, or otherwise does not necessarily constitute or imply its endorsement, recommendation, or favoring by the United States Government or any agency thereof. The views and opinions of authors expressed herein do not necessarily state or reflect those of the United States Government or any agency thereof. 